\title{
Influence of Strong Acid Hydrolysis Processing on the Thermal Stability and Crystallinity of Cellulose Isolated from Wheat Straw
}

\author{
Chemar J. Huntley, ${ }^{1}$ Kristy D. Crews, ${ }^{1}$ Mohamed A. Abdalla, ${ }^{2}$ \\ Albert E. Russell, ${ }^{2}$ and Michael L. Curry ${ }^{1,2}$ \\ ${ }^{1}$ Department of Materials Science and Engineering, Tuskegee University, Tuskegee, AL 36088, USA \\ ${ }^{2}$ Department of Chemistry, Tuskegee University, Tuskegee, AL 36088, USA \\ Correspondence should be addressed to Michael L. Curry; currym@mytu.tuskegee.edu
}

Received 24 November 2014; Revised 17 March 2015; Accepted 21 March 2015

Academic Editor: Jerzy Bałdyga

Copyright (C) 2015 Chemar J. Huntley et al. This is an open access article distributed under the Creative Commons Attribution License, which permits unrestricted use, distribution, and reproduction in any medium, provided the original work is properly cited.

\begin{abstract}
Cellulose extractions from wheat straw via hydrochloric, nitric, and sulfuric acid hydrolysis methods were carried out. X-ray diffraction spectral analyses reveal that depending on the acid conditions used the structure of the cellulose exhibited a mixture of polymorphs (i.e., CI and CIII cellulose phases). In addition, the percent crystallinity, diameter, and length of the cellulose fibers varied tremendously as determined by X-ray diffraction and scanning electron microscopy. Thermal gravimetric analysis measurements revealed that the thermal stability of the extracted cellulose varied as a function of the acid strength and conditions used. Scanning electron microscopy analysis revealed that the aggregation of cellulose fibers during the drying process is strongly dependent upon the drying process and strength of the acids used.
\end{abstract}

\section{Introduction}

Interest in the biodegradability of natural products for use in the production of green electronics and alternative transportation fuels has rapidly increased over the years with a special focus on crystalline cellulose-based composites. Furthermore, with an increase in usage and discarding of technological devices, increasing demand for energy efficient systems, and due to the potential polymeric materials stored within the cellulose structure, cellulose extraction has become a top focus in research labs globally [1-9]. To that end, given the abundance of agricultural waste generated globally, using these products as biomass sources for cellulose extraction is conceivable. In addition to adding to the sources of cellulose for usage in green technology and in alternative fuel production this process could lead to an avenue for reducing the amount of environmental waste generated in landfills. Wheat straw is an agricultural waste that is generated by the ton globally each year and, due to its food chain exemption, would make an excellent biomass source for extraction of cellulosic materials.
Cellulose is an abundant, high molecular weight natural polymer that possesses great strength and biodegradability. Cellulose makes up about 75 percent or more of plant material and is a derivative of crops, plants, and wood [8]. The cellulose chain adopts a linear structural arrangement and, due to its intra- and intermolecular hydrogen bonding, various ordered crystalline arrangements are observed. Furthermore, due to cellulose encasement between lignin and hemicelluloses, the extraction of crystalline cellulose will depend on the method and biomass source used during the extraction process. Processing methods may limit the use or alter the structural integrity of the cellulose. In general, cellulose extracted from plant materials contains both an amorphous region and a crystalline region. It has been reported that crystalline cellulose can exist within four different allomorphs, CI, CII, CIII, and CIV [2,7]. Typically, CI is the most abundant phase and the most sought after due to its optimal elastic properties [3].

The extraction and functionalization of cellulose without introducing structural rearrangements is not a trivial process. 
In fact, depending on the extraction methodology used, cellulose crystal sizes and orientation can be dramatically altered, that is, texturing $[1,6,9]$. Many reports indicate that when using sulfuric acid to extract cellulose, concentrations between $45-65 \mathrm{wt} \%$ yield high crystallinity cellulose particles $[4,5,10]$ while concentrations greater than $65 \mathrm{wt} \%$ caused cellulose swelling and structural degradation [11-13]. Other literature reports indicate that sulfuric acid concentrations around $63 \mathrm{wt} \%$ are capable of breaking hydrogen bonds and forming different complexes such as the partial esterification of hydroxyl groups and, subsequently, their substitution by sulfate groups [14-16]. Although many of these reports are similar in nature the extraction of cellulose using concentrated sulfuric acid hydrolysis remains somewhat ambiguous. It is known that the use of strong mineral acids for chemical hydrolysis introduces cellulose degradation that dehydrates the polymer $[17,18]$. Camacho et al. demonstrated the use of strong acids present at room or lower temperatures, such as sulfuric acid, at concentrations greater than $62-63 \mathrm{wt} \%$ to cause dissolvability and swelling of cellulose [19]. Therefore, higher concentrations hinder the regeneration of cellulose by water dilution, which is due to fast acidic depolymerization, and results in the formation of water-soluble oligomers [20]. Such activation techniques efficiently open and enlarge existing capillaries, pores, or voids, disintegrate fibrillar aggregation, and affect the crystal polymorphs, crystallinity index, and thermal stability [20, 21].

It is well documented that the main factors contributing to the dissolution of cellulose in concentrated sulfuric acid are hydrogen bond breaking due to complex formations and the depolymerization of macromolecular chains. However, there are other factors (e.g., biomass types) that can yield cellulose with drastically different structural and morphological arrangements [6]. Hence, in order to further understand the effects of acid treatment processing of different biomass sources, more systematic investigations comparing different acid treatment processes are necessary to resolve the dependency of the cellulose structure on the parameters used during the acid extraction process. Thus, the aim of this study is twofold: to systematically investigate the dependency of the cellulose structure on the hydrolysis method used (strong versus weak acid hydrolysis extractions) and to investigate the use of wheat straw as a biorenewable source for the generation of crystalline cellulose that can be used as high-quality fillers for composites and strengthening additives.

\section{Experimental}

2.1. Materials. ACS reagent grade chemicals purchased from Sigma-Aldrich were used as received. Acetic acid, acetone, hydrochloric acid, nitric acid, sodium chlorite, sodium hydroxide, and sulfuric acid for cellulose isolation were purchased from Sigma-Aldrich. Wheat straw was purchased from Georgia.

2.2. Isolation of Crystalline Cellulose. Figure 1 shows a detailed account of the extraction methodology used in the separation of crystalline cellulose from wheat straw for

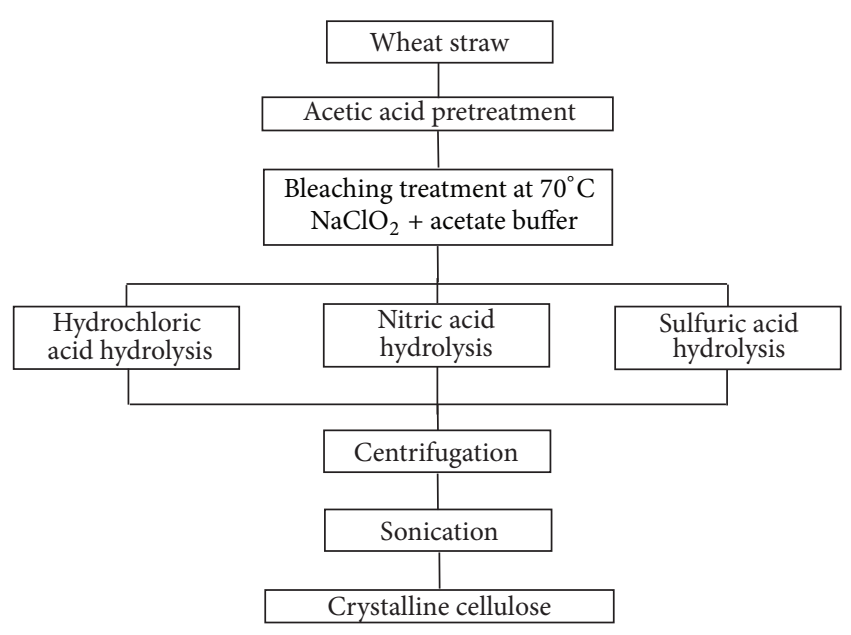

FIGURE 1: Showing the synthesis methodology for the extraction of crystalline cellulose from wheat straw.

comparative analysis. In short, acid solutions were prepared in the following concentrations: 90 weight percent (wt $\%$ ) acetic acid, $9 \mathrm{wt} \%$ sodium chlorite, $58 \mathrm{wt} \%$ acetic acid and $42 \mathrm{wt} \%$ sodium hydroxide buffer solution, 32 volume percent (vol\%) hydrochloric acid, 32 vol\% nitric acid, and 32 vol\% sulfuric acid. Wheat straw was subjected to fractionation by refluxing in the presence of an acetic acid solution for 2 hours. Subsequently, the mixture was filtered using vacuum filtration with Whatman grade 1 filter paper and washed with hot distilled water to rid the wheat straw of residual acid. Bleaching was carried out using a sodium chlorite solution in an acetic acid and sodium hydroxide buffer system at approximately $70^{\circ} \mathrm{C}$ for 2 hours, producing a strong oxidizing agent, chlorous acid. The product was filtered using the previously stated filtration method. The resulting cellulose was treated with the $32 \mathrm{wt} \%$ strong acid solutions-hydrochloric, nitric, or sulfuric acids. The reaction time was 24 hours for each acid. Subsequently, the resulting crystalline cellulose was centrifuged 3 to 6 times at $1500 \mathrm{rpm}$ for 30 minutes and fresh distilled water was added after each centrifugation step. The resulting product was sonicated on an ice bath for 4-8 hours using a Fisher Scientific Ultrasonic Cleaner with operating ultrasonic frequency of $40 \mathrm{KHz}$, centrifuged three additional times (using prior centrifugation conditions), and allowed to settle for 24 hours. The distilled water was decanted from the product and replaced with acetone using the solvent exchange methodology. Further centrifugation was performed at 1000 RPM for 10 minutes and, afterwards, fresh acetone was added. The process was repeated for 3 to 4 times and allowed to settle for 24 hours. Subsequently, all cellulose extracts are dried under vacuum at $70^{\circ} \mathrm{C}$ for 24 hours in a Thermo-Scientific Lab-Line vacuum oven and converted into powder form using a mortar and pestle for further analysis.

\subsection{Characterization of Crystalline Cellulose}

2.3.1. X-Ray Diffraction Spectroscopy. X-ray diffraction (XRD) was used to determine the crystallographic structure 
of cellulose crystal extracted from wheat straw. After drying, $\mathrm{X}$-ray diffraction analyses were carried out on samples using a Rigaku D/MAX 2200 X-ray diffractometer with a diffracted beam graphite monochromatic running on $\mathrm{Cu} K \alpha$ radiation. Samples of the cellulose powder are placed on a glass slide and analyses are performed from $0^{\circ}$ to $80^{\circ}$ of $2 \theta$ angle at a rate of 5 degrees per minute. The data was collected and peaks were analyzed using PDF database of Joint Committee on Powder Diffraction Standards (JCPDS) and reported literature findings for crystalline cellulose.

XRD calculated cellulose patterns were produced using Mercury 3.3 programming software. The.cif files for $\mathrm{CI}_{\alpha}$, $\mathrm{CI}_{\beta}$, and $\mathrm{CIII}_{(\mathrm{I})}$ were downloaded from the supplemental materials hosted on the SpringerLink website. Since it is well known that both the $\mathrm{CI}_{\beta}$ and the $\mathrm{CI}_{\alpha}$ polymorphs are extracted under hydrolysis conditions, an average spectra was obtained "CI" and used to generate an average pattern demonstrating the mix of $\mathrm{CI}$ and $\mathrm{CIII}_{(\mathrm{I})}$ polymorphs. The peak at half maximum for the experimental XRD data was used to determine the simulated crystal size, and the composition of the mixed polymorphs was set at $55 \% \mathrm{CIII}_{(\mathrm{I})}$ crystals and $45 \%$ CI crystals. Subsequently, the experimental XRD diffraction pattern was overlaid atop the calculated XRD theoretical pattern for comparative analysis.

2.3.2. Scanning Electron Microscopy. Scanning electron microscopy (SEM) imaging of crystalline cellulose was carried out using a Zeiss EVO 50VP scanning electron microscope. The crystalline cellulose was analyzed by sputter coating with gold using a 550X Sputter Coating device. The Zeiss EVO 50VP was operated at an acceleration voltage of $20 \mathrm{kV}$. For each sample, different parts of the grid were used to determine both average shape and size distributions.

2.3.3. Thermogravimetric Analysis. Thermal stability of the cellulose extract was determined using a TA Instrument Q500 Thermogravimetric Analyzer (TGA). Analysis was performed on samples of $10-15 \mathrm{mg}$ in an oxygen atmosphere from $30^{\circ} \mathrm{C}$ to $550^{\circ} \mathrm{C}$ at a heating rate of $5^{\circ} \mathrm{C} /$ minute.

\section{Results and Discussion}

Acidic pretreatment, or hemicellulose hydrolysis, involves the use of an acid or hot water conditions to breakdown the primary cell wall to expose the underlying cellulose, hemicellulose, and lignin, shown in Scheme 1. The removal of the hemicellulose and lignin is generally done by an acid or base due to its inexpensiveness [22]. Reports suggest that diluted acids in appropriate temperature parameters are known to effectively remove hemicellulose, increasing the cellulosic yield [23]. However, further treatment procedures, such as bleaching or chemical pulping, are undergone to release the encased cellulose. Hydrolysis techniques, or an activation method [24], tend to remove the amorphous regions, producing glucose monomers $[18,25]$. The acid specifically targets the amorphous regions, due to the disorder and voids present there, whereas the more ordered regions tend to lack these voids [20].
3.1. X-Ray Diffraction Analysis. XRD was used to study the resulting crystal structure (polymorph) of cellulose crystallites formed and the percent crystallinity of cellulose generated during the different acid hydrolysis treatment processes. Figures 2(a)-2(d) display typical XRD patterns of commercial microcrystalline cellulose (cMCC) and cellulose extracted (EC) from wheat straw using acid hydrolysis $\left(\mathrm{HCl}, \mathrm{HNO}_{3}\right.$, and $\mathrm{H}_{2} \mathrm{SO}_{4}$, Figures 2(b)-2(d), resp.). As can be observed in the XRD spectra in Figures 2(a)-2(d), three major peaks are evident for both the $\mathrm{CMCC}$ and the extracted cellulose. These peaks are located around $15^{\circ}, 22.5^{\circ}$, and $35^{\circ} 2 \theta$ and, based on XRD studies on the structure of cellulose $\mathrm{I}_{\beta}$ by

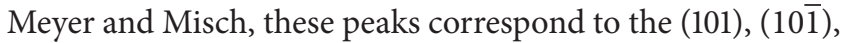
(002), and (040) diffraction planes, respectively [26, 27]. However, in the extracted cellulose XRD spectra, Figures 2(b)-2(d), the observance of additional low intensity XRD peaks can be seen at $2 \theta=7.5^{\circ}$ and $11.7^{\circ}$. Based on reported literature, the diffraction peak at $11.7^{\circ} 2 \theta$ is representative of the CIII cellulose polymorph [2]. This is suggestive of a mixture of the CI and CIII cellulose polymorphs extracted from wheat straw using the acid hydrolysis method. Furthermore, in this case, a new crystal (101) diffraction plane is evident. In addition, the (10 $\overline{1})$ plane typically appears at a diffraction angle of $20.6^{\circ} 2 \theta$ for the CIII phase; however, given that the broad, dominant (002) peak for the CI phase appears at a diffraction angle around $22^{\circ} 2 \theta$, this peak could be masked from identification without using specific XRD peak deconvolution software. Given the three hydrolysis processes, the low intensity peaks are more pronounced in the case of acid hydrolysis of the cellulose structure under sulfuric acid conditions, Figure 2(c). Reports by Ioelovich indicated that, at sulfuric acid concentrations greater than 63$64 \mathrm{wt} . \%$, portions of the cellulose will swell and dissolve and, with concentrations between 64-65 wt.\% under controlled conditions, the regenerated dissolved cellulose exhibits a CII polymorph structural arrangement [20]. That is, the insoluble cellulose exhibits a crystalline structure CI while the regenerated cellulose exhibits a crystalline polymorph CII. This further supports our findings of a CI/CIII mixture since the regeneration process is occurring here as well.

To further investigate the observed mixture of CI and CIII polymorphs upon acid hydrolysis, X-ray diffraction patterns of the CI and CIII polymorphs were generated using a method reported by French [26]. In this analysis, Mercury 3.3 programming software was used to generate diffraction patterns for both the $\mathrm{CI}_{\beta}$ and $\mathrm{CI}_{\alpha}$ and $\mathrm{CIII}_{(\mathrm{I})}$ polymorphs. For spectra clarity during overlapping and since no peaks appear beyond that of $50^{\circ} 2 \theta, \mathrm{XRD}$ spectra for the calculated and experimental extracted cellulose were compared between the angles from $0^{\circ}$ to $50^{\circ} 2 \theta$. The $\mathrm{CI}_{\beta}$ and $\mathrm{CI}_{\alpha}$ and $\mathrm{CIII}_{(\mathrm{I})}$. cif files were downloaded from the supplemental materials hosted on the SpringerLink website as indicated by French [26]. Using excel, the resulting diffraction patterns were mixed to deduce a CI-CIII ${ }_{(\mathrm{I})}$ diffraction pattern. Figures 3(a)-3(c) show the calculated diffraction pattern for mixed cellulose $\mathrm{CI}_{\beta}$ and $\mathrm{CI}_{\alpha}(\mathrm{CI}), \mathrm{CIII}_{(\mathrm{I})}$, and a mixture of $\mathrm{CI}$ and $\mathrm{CIII}(\mathrm{I})$ polymorphs. The calculated mixed CI-CIII $(\mathrm{I})$ XRD patterns are overlaid with a typical XRD spectrum for cellulose 

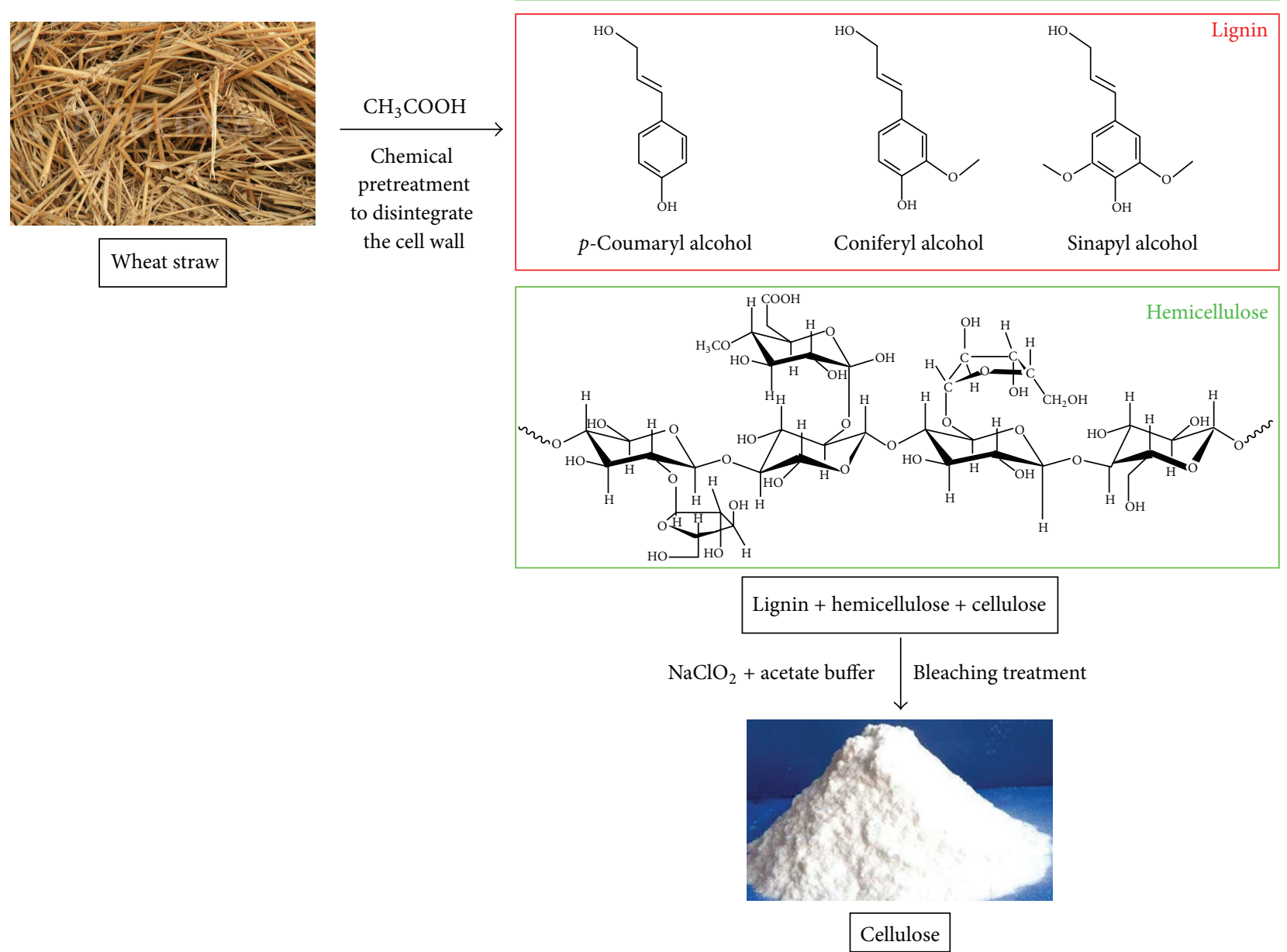

SCHEME 1: Revealing the acid hydrolysis treatment processing and structural changes.

extracted using sulfuric acid hydrolysis methodology for pattern comparison. In the calculated patterns case, the peaks are generated assuming perfect crystals; thus, peak broadening due to variations in crystal sizes and background level due to amorphous cellulose regions are not observed. However, based on the calculated diffraction peaks shown in Figures 3(a) and 3(b), cellulose $\mathrm{CI}$ and $\mathrm{CIII}_{(\mathrm{I})}$ polymorphs display three main diffraction peaks at $2 \theta=14.8^{\circ}, 16.6^{\circ}$, and $22.9^{\circ}$ and at $2 \theta=11.6^{\circ}, 17.2^{\circ}$, and $20.9^{\circ}$, respectively. In addition, the calculated $\mathrm{CIII}_{(\mathrm{I})}$ diffraction pattern also reveals a shoulder peak at $20.6^{\circ} 2 \theta$ polymorph; see Figure $3(\mathrm{~b})$. Mixing of the calculated XRD patterns for cellulose $\mathrm{I}_{\beta}$ and cellulose $\mathrm{III}_{(\mathrm{I})}$ polymorphs reveals a new XRD pattern that is similar to the experimental pattern obtained for the acid hydrolysis of cellulose from wheat straw. Figure 3(c) shows the XRD average patterns for the calculated mixed cellulose
$\mathrm{I}_{\beta}$ and $\mathrm{III}_{(\mathrm{I})}$ and the experimentally extracted cellulose from wheat straw. In the calculated patterns of the mixed cellulose polymorphs, several main peaks are observed at $2 \theta=11.6^{\circ}$, $14.7^{\circ}, 16.6^{\circ}, 17.1^{\circ}, 20.6^{\circ}, 20.9^{\circ}$, and $22.9^{\circ}$, which match well with the overlapping experimental XRD data for extracted cellulose. This future proves that the appearance of the peak at $11.6^{\circ} 2 \theta$, based on the generated patterns, is clearly indicative of the (010) diffraction plane of $\mathrm{CIII}_{(\mathrm{I})}$ polymorph. Furthermore, this provides more evidence of mixed cellulose $\mathrm{I}_{\beta}$ and $\mathrm{III}_{(\mathrm{I})}$ crystal structures upon extraction from wheat straw using strong acid hydrolysis. Although mixing of cellulose $\mathrm{I}_{\beta}$ and $\mathrm{III}_{(\mathrm{I})}$ polymorphs appears in all three acid treatments, it is more prevalent in the sulfuric acid samples. Thus, in this case, it is conceivable that the hydrolysis time and acid strengths play a key role in the observance of the mixed cellulose polymorphs. 


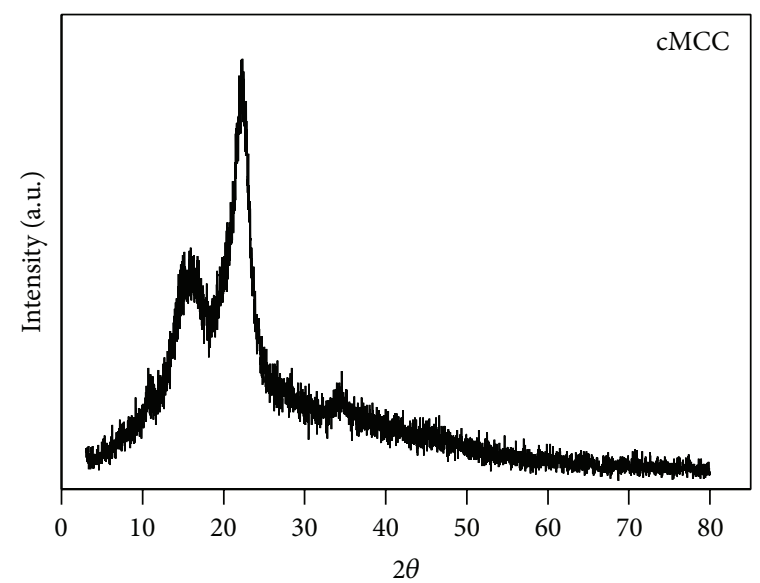

(a)

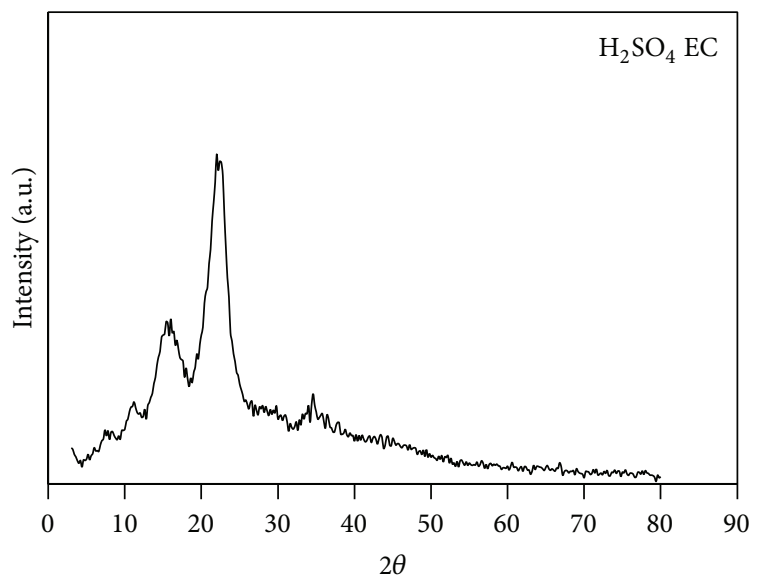

(c)

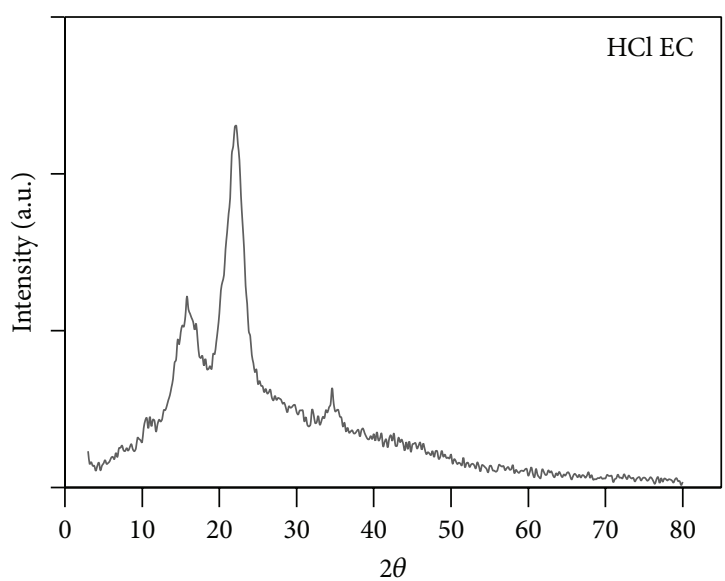

(b)

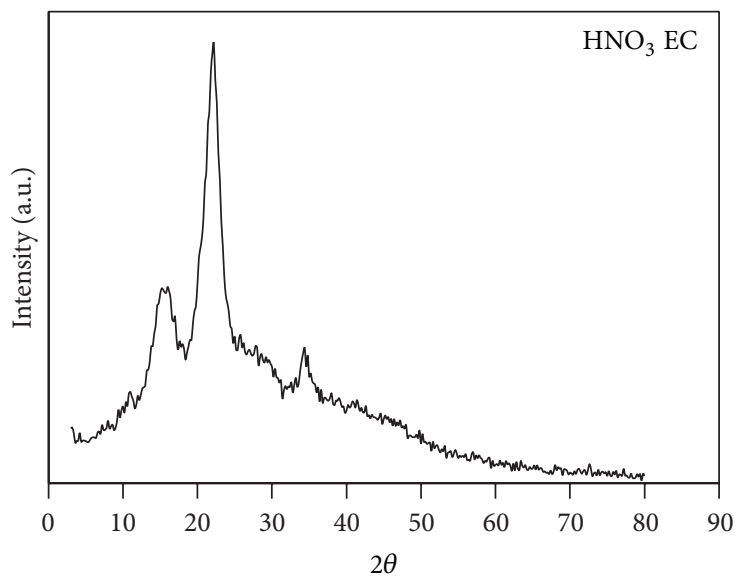

(d)

FIGURE 2: Showing the X-ray diffraction analyses of commercial cellulose (a) and acid hydrolyzed cellulose from wheat straw ((b)-(d)).

Apparently the observance of the XRD peak located at $7.5^{\circ}$ $2 \theta$ for the experimental data is absent in the calculated XRD pattern. However, literature reports by Klemm and coworkers indicate that depending on extraction conditions it is possible to convert cellulose I into many different alkali forms with different crystal structures and variable $\mathrm{NaOH}$ and water content [27]. In addition, it has been observed that sodium cellulose I exhibits a peak around $7.5^{\circ} 2 \theta$. Hence, given that sodium hydroxide is used in the pretreatment method, it is possible during the incomplete conversion of cellulose $\mathrm{I}_{\beta}$ and cellulose III $(\mathrm{I})$ that sodium cellulose I exists within the polymorph mixture.

The percent crystallinity of the cellulose extract was determined with the assumption that the $\mathrm{CI}_{\beta}$ crystal structure is the dominant phase present based on the appearance of the highest intensity peak being assigned to the (002) plane. Equation (1) was used to perform the percent crystallinity of the cMCC and cellulose extract from the different acid hydrolysis processes:

$$
\% \text { Crystallinity }=\frac{I_{\text {crystalline }}}{I_{\text {amorphous }}+I_{\text {crystalline }}} \times 100 \% \text {, }
$$

TABLE 1: Revealing cellulose particle sizes and percent crystallinity for $\mathrm{CMCC}$ and EC.

\begin{tabular}{lccc}
\hline $\begin{array}{l}\text { Extraction } \\
\text { process }\end{array}$ & $\begin{array}{c}\text { Average } \\
\text { length }(\mu \mathrm{m})\end{array}$ & $\begin{array}{c}\text { Average } \\
\text { width }(\mu \mathrm{m})\end{array}$ & $\begin{array}{c}\text { Crystallinity } \\
(\%)\end{array}$ \\
\hline $\begin{array}{l}\text { Commercial } \\
\text { Hydrochloric }\end{array}$ & 100.35 & 9.317 & 69.29 \\
acid & 145.93 & 32.10 & 67.35 \\
Nitric acid & 218.63 & 18.70 & 71.88 \\
Sulfuric acid & 114.16 & 12.06 & 70.58 \\
\hline
\end{tabular}

where $I_{\text {crystalline }}$ corresponds to the (002) crystalline peak located at a $2 \theta$ of $22.5^{\circ}$ and $I_{\text {amorphous }}\left(I_{\mathrm{AM}}\right)$ is the highest peak of the amorphous background region located at a $2 \theta$ around $18^{\circ}$ [28]. Table 1 shows the different percent crystallinity values determined for $\mathrm{CMCC}$ and cellulose extracted using the nitric, sulfuric, and hydrochloric acid hydrolysis process. The percent crystallinity for the cellulose extracted from wheat straw is slightly higher for the nitric and sulfuric acid cases when compared to the cMCC (69.29 versus 71.88 and 70.58 , resp.). The percent crystallinity for the hydrochloric acid case is lower than the cMCC and nitric and sulfuric extracted 


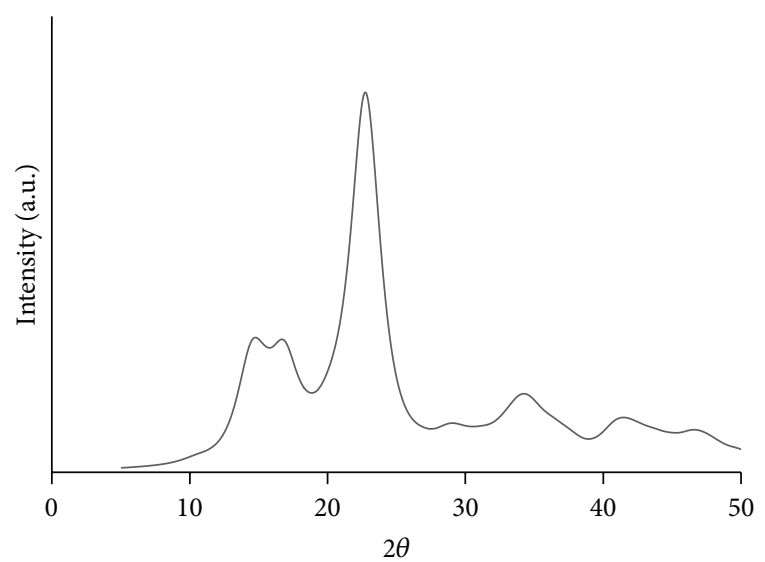

(a)

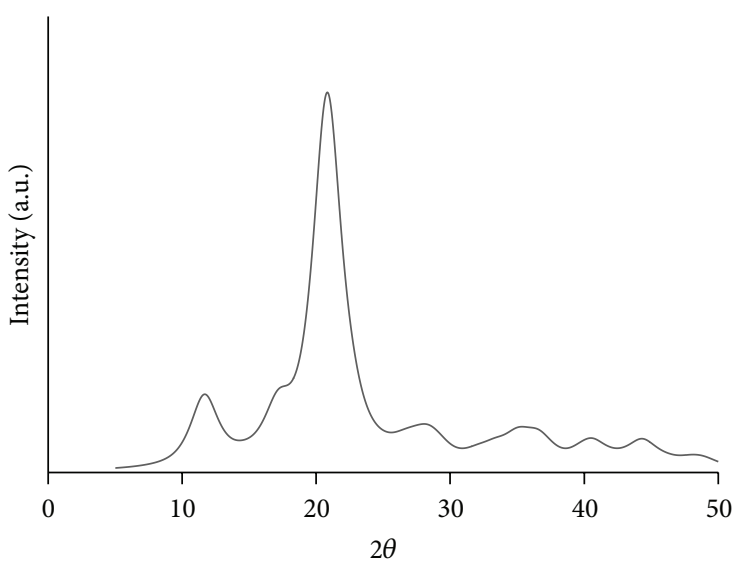

(b)

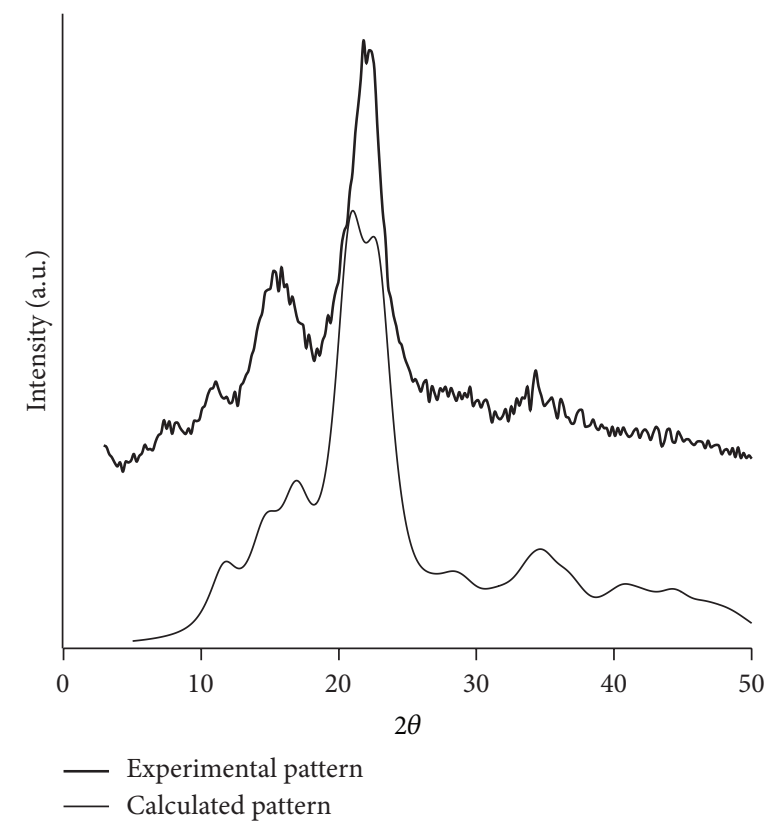

(c)

FIGURE 3: Revealing calculated patterns for $\mathrm{CI}_{\beta}$ and $\mathrm{CIII}_{(\mathrm{I})}((\mathrm{a})-(\mathrm{b}))$ and mixed patterns of $\mathrm{CI}_{\beta}$ and $\mathrm{CIII}_{(\mathrm{I})}$ with experimentally obtained XRD patterns (c).

cellulose. It is speculated here that the slight changes in percent crystallinity for the acid extractions are directly related to the acid strengths, since the hydrolysis times were constant. It has been shown that, at high concentrations, sulfuric acid is capable of breaking hydrogen bonds, which allows penetration into amorphous and crystalline cellulose regions [14-17, 20]. Based on $\mathrm{pKa}$ values, the order of acid strength is as follows: $\mathrm{HCl}>\mathrm{H}_{2} \mathrm{SO}_{4}>\mathrm{HNO}_{3}$ (all having $\mathrm{pKa}$ values $<1)$. As revealed through Table 1 , as the strength of the acid increases $\left(\mathrm{HCl}>\mathrm{H}_{2} \mathrm{SO}_{4}>\mathrm{HNO}_{3}\right)$ the percent crystallinity decreases $\left(\mathrm{HCl}<\mathrm{H}_{2} \mathrm{SO}_{4}<\mathrm{HNO}_{3}\right)$. This may be rationalized through the increased breaking of hydrogen bonds as the strength of the acid increases, which allows for increased degradation of the amorphous and crystalline regions.
3.2. Scanning Electron Microscopy Analysis. Typically, microcrystalline cellulose is porous with diameters, or widths, that range between $10-50 \mu \mathrm{m}$ and has high crystallinity values and aggregates of multisized cellulose crystals due to the strong hydrogen bonding [29]. Similar results are observed here for cMCC. The cMCC has a typical average diameter and crystallinity value that is in agreement with literature reports (ca. $13 \mu \mathrm{m}$ and $69 \%$, resp.). Figures 4 (a)4(e) reveal the surface morphology, shape, and sizes for cMCC and cellulose extracted using nitric, sulfuric, and hydrochloric acids. In this analysis, the lengths of several cellulose fibers were measured to generate an average error associated with their mean measured values. Apparently, cMCC cellulose exhibits similar widths and lengths to that of the microcrystalline cellulose (see Table 1). The cMCC 


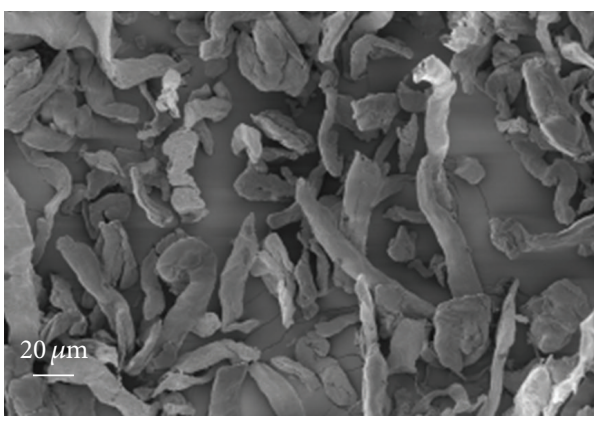

(a)

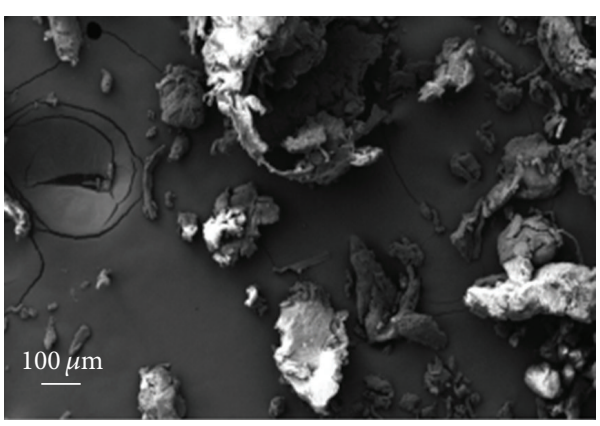

(c)

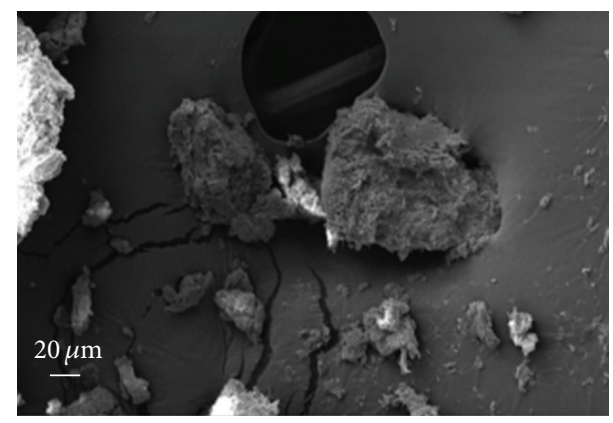

(b)

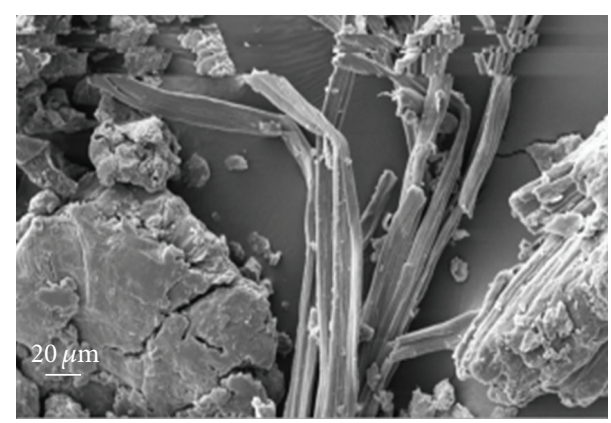

(d)

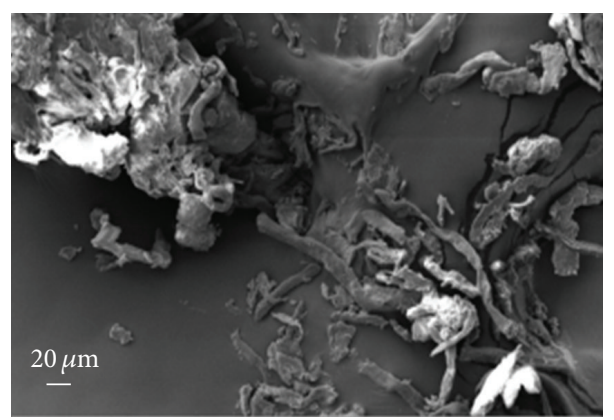

(e)

FIGURE 4: Revealing SEM images of commercial cellulose, cMCC (a), lignocellulose (b), hydrochloric (c), nitric (d), and sulfuric (e) cellulose.

cellulose crystals are well dispersed in sizes with an apparent rod-like shaped confirmation indicating the formation of cellulose microcrystals; see Figure 4(a).

It is well known that smaller well-defined fibers are typically at the top of your acid hydrolysis solutions but, in this case, the authors have focused on the bulk of the materials to examine the drying effect occurring within the three extraction processes. Hence, similar crystallinity values, shapes, and diameters are observed for EC under the different acid conditions; see Figures 4(b)-4(e). Cellulose extracted using the nitric and sulfuric acid methods exhibits similar rod-like shapes when compared to the cMCC; however, the length and width of the EC exhibit much larger values when compared to the cMCC. In the case of nitric acid, both rod-like shaped fibers and shorter rodlike microcrystal structures can be observed; see Figure 4(d). It is well documented that cellulose extracted from plants exhibits the largest of the particle types [6]. Similar results are observed for the sulfuric acid extraction of cellulose
(Figure 4(e)). Furthermore, cellulose aggregation is observed for both nitric and sulfuric acid methods. One may speculate that the aggregation is occurring due to incomplete removal of impurities during the pretreatment process. Figure 4(b) reveals an SEM of cellulose extracted with residual impurities from the pretreatment process. As can be observed, under these conditions, no cellulose fibers are produced and only large-scale aggregation is observed. Many reports indicated that depending on the method of filtration and drying of the cellulose particles, aggregation could be initiated. In fact, several reports in the literature noted that depending on the acid hydrolysis process used, variability in shapes and sizes are not consistent from extraction to extraction and can induce agglomeration of smaller cellulose fibers into larger fibers with various geometries [29-31]. Hence the stages of drying of cellulose are threefold-(1) a constant ratedrying period, (2) the first falling rate-drying period, and (3) the second falling rate-drying period [32-35]. In stage 1 , water begins to evaporate, causing cellulose particles to 
begin diffusing closer together and eventually exposing the polymer's surface enabling surface water evaporation (the first falling rate-drying period). As a result, surface tension occurs due to the water molecules present on the surface of cellulose commanding the initiation of vapor diffusion and the particles moving closer together. The third phasethe second falling rate-drying period-begins as the interior water-transferring rate becomes smaller than the surface diffusion rate, developing full contact of the cellulose and a hydrogen-bonded network $[36,37]$. To that end, depending on the conditions during extraction and drying of the EC, the typical reported length or morphological geometry may not be achieved. In this case, we propose that the induced aggregation of the cellulose during the hydrolysis process is a direct result of the drying process.

However, one cannot discount the ability of the acids to deprotonate (i.e., "acid strength") as a contributor to the aggregation effect of cellulose. In the case of $\mathrm{HCl}$, lesser welldefined cellulose fibers or crystals are evident with mostly large scale aggregation occurring. A simple rationale of the strength of the $\mathrm{HCl}$ acid in comparison to $\mathrm{HNO}_{3}$ and $\mathrm{H}_{2} \mathrm{SO}_{4}$ could be provided here as an explanation to this occurrence. That is, given that the hydrolysis time, concentration of the acids, and temperature were held constant and $\mathrm{HCl}$ has a smaller pKa value than $\mathrm{HNO}_{3}$ or $\mathrm{H}_{2} \mathrm{SO}_{4}$, we speculate that hydrolyzation of the cellulose amorphous and crystalline regions could be significantly reduced, which has been reported to alter the structural arrangement of the extracted cellulose. In addition, it is also conceivable that the higher pKa value for nitric and sulfuric acids results in less charge transfer to the cellulose structure (i.e., protonation). That is, the amount of hydrogen observed within the cellulose network will be greater with decreasing $\mathrm{pKa}$ values $(\mathrm{HCl}<$ $\mathrm{H}_{2} \mathrm{SO}_{4}<\mathrm{HNO}_{3}$ ) and this increase will lead to increased aggregation of the cellulose during the drying process via hydrogen bonding.

Among the acid hydrolyses, nitric and sulfuric acid methods prove to be the best methods needed to generate well-dispersed crystalline cellulose with minimal aggregation affects; see Figures 4(d) and 4(e). These particles are rodlike in shapes with diameters and crystallinity values that match well with the cMCC tested here and with reported literature values [29]. However, aggregations of the EC fibers are observed due to processing parameters. To that end, SEM has verified that the EC gives similar properties to those of the cMCC but exhibits some aggregation which is induced by strong hydrogen bonding and processing parameters. Further investigations are currently being carried out on techniques to disrupt the hydrogen bonding to limit the amount of aggregation observed during the hydrolysis process, which will evidently yield more desired cellulose materials.

3.3. Thermogravimetric Analysis. Figure 5 reveals the thermal behavior of cellulose fibers extracted using the various acid hydrolysis methods. TGA curves for cMCC reveals only one decomposition temperature around $300^{\circ} \mathrm{C}$. However, it is clear from Figure 5 that the acid extracted cellulose possesses many decomposition steps. However, cellulose extracted

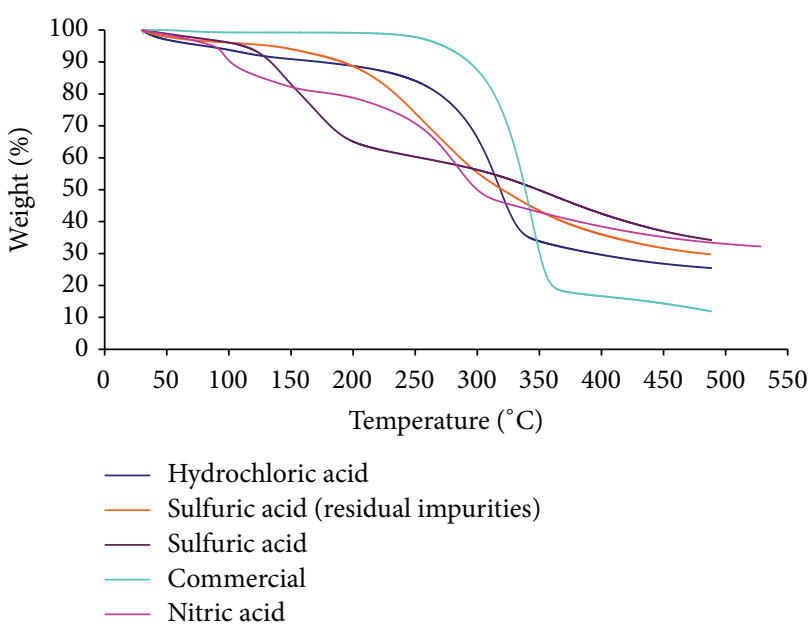

FIGURE 5: Revealing the thermogravimetric analysis curves for cMCC and cellulose extracted using acid hydrolysis methodology.

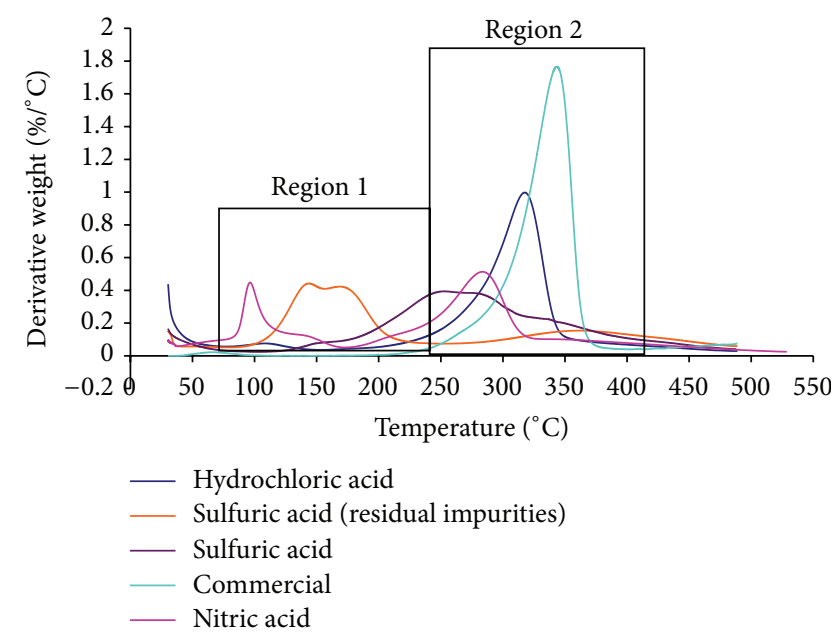

FIGURE 6: Revealing the thermogravimetric analysis derivative curves for cMCC and cellulose extracted using acid hydrolysis methodology.

using sulfuric acid followed by oven-drying and sulfuric acid with residual impurities shows thermal behavior that is quite similar to that of the cMCC. To that end, in an effort to simplify this analysis, two inflection point temperatures (i.e., Region 1 and Region 2, between 50 to 150 and 250 to 400 degrees Celsius, resp.) are used as focal points in this part of the discussion and can be observed in Figure 6. We have concluded that, in the box indicated as Region 1, due to the hydrophilic nature of the cellulose structure, water uptake is being released at this temperature. That is, cellulose is known to swell due to the absorption of water and, upon heating, will dehydrate through the loss of $\mathrm{H}_{2} \mathrm{O}$. This assumption was verified through the preheating of the cellulose samples before analyzing with TGA. No rate of decomposition peak for water in Region 1 is observed for cellulose extracted via sulfuric acid after undergoing an annealing "oven-drying" pretreatment process. 


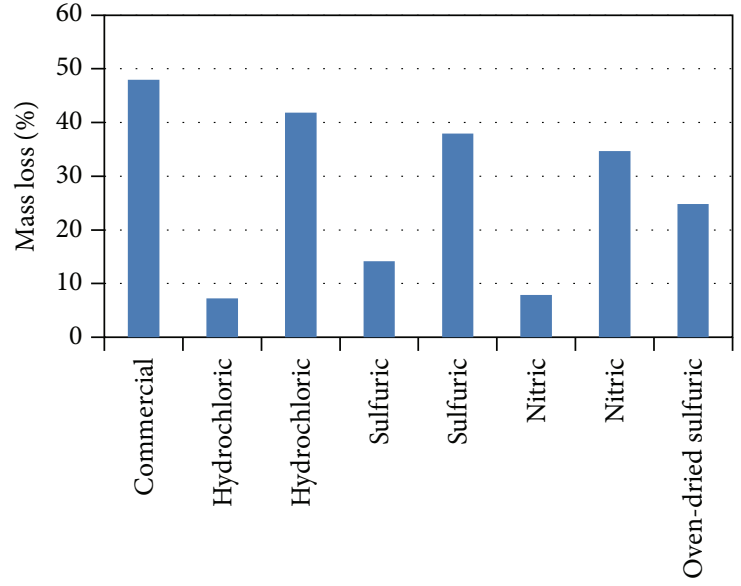

(a)

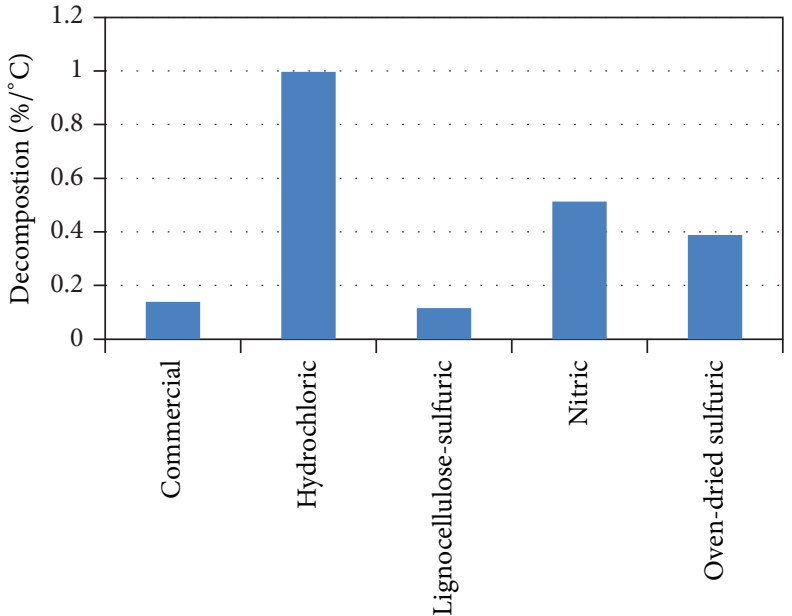

(b)

Figure 7: Showing bar graphs of the total percentage of mass loss during water evaporation and decomposition (a) and rate of mass loss during decomposition (b) for cMCC and cellulose extracted from wheat straw, respectively, using acid hydrolysis methodology.

A clearer picture of the decomposition mechanisms of the cMCC and EC can be seen in Figures 7(a) and 7(b). Figure $7(\mathrm{a})$ is a bar graph representing the amount of mass loss during the release of water $\left(\mathrm{ca} .100^{\circ} \mathrm{C}\right)$ and during the degradation of the cellulose polymer $\left(>200^{\circ} \mathrm{C}\right)$; see Figure 5 . For comparative analysis, Figure $7(\mathrm{~b})$ is a bar graph that represents the rate at which the mass is being lost in Region 2; see Figure 6. Figure 7(a) shows the percentage of mass lost by cMCC and EC under various acid hydrolysis conditions. As stated earlier, water loss due to dehydrating of the cellulose structure occurs around $100^{\circ} \mathrm{C}$. For both the cMCC and the oven-dried EC only one degradation temperature is observed and is located in Region 2 of Figure 6. That is, cMCC and oven-dried EC only show one temperature at which the greatest mass amount of the cellulose structure is lost as indicated by the bar graph. This further verifies that Region 1 (i.e., between 50 to $150^{\circ} \mathrm{C}$ ) is typically where dehydration of the EC occurs. In the case of the cMCC, we speculate that this is a direct result of the cellulose crystal processing before purchasing from the vendor (i.e., curing). However, the EC undergoes several soaking and rinsing cycles where evidently water uptake occurs, as verified through the ovendrying analysis. Based on the second inflection point, Region 2, between 150 to $300^{\circ} \mathrm{C}$, we observed that cMCC has the greatest mass loss $(>47 \%)$ at its second inflection point around 300 degrees Celsius; see Figure 7(a). The ECs show a smaller mass loss $(<42 \%)$ at this temperature when compared to the cMCC. This is apparently indicative of superior thermal stability exhibited by the cellulose extracted under the various acid conditions when compared to the cMCC. This can be explained through the potential substituted groups on the EC skeletal structure. Huang and coworkers reported that an increase in thermal stability was observed for cellulose tristearate (cellulose ester) due to the regular arrangement of the side chains [37]. It is proposed here that based on the acid strength used, structure rearrangement (e.g., cyclization) can be promoted influencing the amount and rate at which the mass is lost.

Furthermore, Figure 7(a), among the acid hydrolyses, reveals that $\mathrm{HCl}$ has the greatest mass loss while the ovendried $\mathrm{H}_{2} \mathrm{SO}_{4}$ loses the least of its mass at the higher temperature range, Region 2. At a closer view, Figure 7(b) reveals the rate at which the mass is lost for $\mathrm{cMCC}$ and acid-treated samples for Region 2. $\mathrm{HCl}$ loses its mass at a much faster rate per degree Celsius than $\mathrm{HNO}_{3}$ or $\mathrm{H}_{2} \mathrm{SO}_{4}$. In addition, $\mathrm{HCl}$ has the least residual mass when compared to $\mathrm{HNO}_{3}$, or $\mathrm{H}_{2} \mathrm{SO}_{4}$, but similar residual mass when compared to the cMCC (see Figure 7(a)). That is, the cellulose extracted from the wheat straw via the $\mathrm{HNO}_{3}$ and $\mathrm{H}_{2} \mathrm{SO}_{4}$ shows greater mass resistance to change at temperatures near $300^{\circ} \mathrm{C}$.

\section{Conclusions}

It has been concluded from this study that the extraction of cellulose from an agricultural waste product such as wheat straw using strong acid hydrolysis such as sulfuric and nitric acids will yield similar crystalline and thermal properties as currently reported in the literature. Hence, in this study, we have demonstrated the effect of various strong acids on the polymeric, structural, and thermal properties of cellulose extracted from wheat straw. Based on cellulose extracted using three different acid hydrolysis methods $\left(\mathrm{HCl}, \mathrm{HNO}_{3}\right.$, and $\mathrm{H}_{2} \mathrm{SO}_{4}$ ), it was found that cellulose particles with similar sizes and crystallinity were obtained. XRD spectra revealed that the extracted cellulose exhibited a mixture of CI and CIII phases with the predominant mixture observed for the $\mathrm{H}_{2} \mathrm{SO}_{4}$ extraction methodology. As calculated using (1), it was found that the percent crystallinities of the $\mathrm{HNO}_{3}$ and $\mathrm{H}_{2} \mathrm{SO}_{4}$ extracted cellulose were slightly higher than those of the purchased cellulose. The $\mathrm{HCl}$ showed a similar percent crystallinity when compared to the cMCC. Given that the higher crystallinity for the cellulose extract was observed 
for the weaker acids $\left(\mathrm{HNO}_{3}\right.$ and $\left.\mathrm{H}_{2} \mathrm{SO}_{4}\right)$, we conclude that, in cases where increased crystallinity is needed, the use of weaker acids would be beneficial in obtaining cellulose crystals with specific properties. Furthermore, SEM revealed that the cellulose particles exhibited average widths and lengths on the micron-level with rod-shapes as the preferred geometry. In addition, depending on the drying and lignin removal processing, aggregation was observed. Moreover, TGA analysis revealed that, in comparison to the commercial cellulose, $\mathrm{HNO}_{3}$ and $\mathrm{H}_{2} \mathrm{SO}_{4}$ cellulose extracts showed the greatest mass resistance to change at temperatures near or above $300^{\circ} \mathrm{C}$.

\section{Conflict of Interests}

The authors declare that there is no conflict of interests regarding the publication of this paper.

\section{Acknowledgments}

The authors gratefully acknowledge the National Science Foundation under Grant nos. NSF EPS-1158862, NSF HRD1137681, and NSF IGERT on Sustainable Electronics DGE1144843 for support of this research. The Department of Chemistry and Chemistry Center for Synthesis and Characterization are recognized.

\section{References}

[1] X. Cao, Y. Chen, P. R. Chang, A. D. Muir, and G. Falk, "Starchbased nanocomposites reinforced with flax cellulose nanocrystals," Express Polymer Letters, vol. 2, no. 7, pp. 502-510, 2008.

[2] E. N. Johnson Ford, S. K. Mendon, S. F. Thames, and J. W. Rawlins, "X-ray diffraction of cotton treated with neutralized vegetable oil-based macromolecular crosslinkers," Journal of Engineered Fibers and Fabrics, vol. 5, no. 1, pp. 10-20, 2010.

[3] A. Ishikawa and T. Okano, "Fine structure and tensile properties of ramie fibres in the crystalline form of cellulose I, II, IIII and IVI," Polymer, vol. 38, no. 2, pp. 463-468, 1997.

[4] D. Y. Liu, X. W. Yuan, D. Bhattacharyya, and A. J. Easteal, "Characterisation of solution cast cellulose nanofibre-reinforced poly(lactic acid)," Express Polymer Letters, vol. 4, no. 1, pp. 26-31, 2010.

[5] L. Ludueña, D. Fasce, V. A. Alvarez, and P. M. Stefani, "Nanocellulose from rice husk following alkaline treatment to remove silica," BioResources, vol. 6, no. 2, pp. 1440-1453, 2011.

[6] R. J. Moon, A. Martini, J. Nairn, J. Simonsen, and J. Youngblood, "Cellulose nanomaterials review: structure, properties and nanocomposites," Chemical Society Reviews, vol. 40, no. 7, pp. 3941-3994, 2011.

[7] A. C. O'Sullivan, "Cellulose: the structure slowly unravels," Cellulose, vol. 4, no. 3, pp. 173-207, 1997.

[8] V. K. Varshney and S. Naithani, "Chemical functionalization of cellulose derived from nonconventional sources," in Cellulose Fibers: Bio- and Nano-Polymer Composites-Green Chemistry Technology, S. Kalia, B. S. Kaith, and I. Kaur, Eds., vol. 19, pp. 43-60, Springer, Berlin, Germany, 2011.

[9] S. Wang and Q. Cheng, "A novel process to isolate fibrils from cellulose fibers by high-intensity ultrasonication, part 1: process optimization," Journal of Applied Polymer Science, vol. 113, no. 2, pp. 1270-1275, 2009.

[10] J. I. Morán, V. A. Alvarez, V. P. Cyras, and A. Vázquez, "Extraction of cellulose and preparation of nanocellulose from sisal fibers," Cellulose, vol. 15, no. 1, pp. 149-159, 2008.

[11] S. Elazzouzi-Hafraoui, Y. Nishiyama, J.-L. Putaux, L. Heux, F. Dubreuil, and C. Rochas, "The shape and size distribution of crystalline nanoparticles prepared by acid hydrolysis of native cellulose," Biomacromolecules, vol. 9, no. 1, pp. 57-65, 2008.

[12] G. Siqueira, J. Bras, and A. Dufresne, "Luffa cylindrica as a lignocellulosic source of fiber, microfibrillated cellulose, and cellulose nanocrystals," BioResources, vol. 5, no. 2, pp. 727-740, 2010.

[13] D. Bondeson, A. Mathew, and K. Oksman, "Optimization of the isolation of nanocrystals from microcrystalline cellulose by acid hydrolysis," Cellulose, vol. 13, no. 2, pp. 171-180, 2006.

[14] I. Z. Selim, A. A. F. Zikry, and S. H. Gaber, "Physicochemical properties of prepared cellulose sulfates: II. From linen pulp bleached by the $\mathrm{H}_{2} \mathrm{O}_{2}$ method," Polymer-Plastics Technology and Engineering, vol. 43, no. 5, pp. 1387-1402, 2004.

[15] N. A. Vedernikov and V. K. Kalnina, Wood Cell Wall and Its Changes at Chemical Treatments, Chemistry, Riga, Latvia, 1972.

[16] M. Roman and W. T. Winter, "Effect of sulfate groups from sulfuric acid hydrolysis on the thermal degradation behavior of bacterial cellulose," Biomacromolecules, vol. 5, no. 5, pp. 16711677, 2004.

[17] Y. Sun and J. Cheng, "Hydrolysis of lignocellulosic materials for ethanol production: a review," Bioresource Technology, vol. 83, no. 1, pp. 1-11, 2002.

[18] C. Li and Z. K. Zhao, "Efficient acid-catalyzed hydrolysis of cellulose in ionic liquid," Advanced Synthesis \& Catalysis, vol. 349, no. 11-12, pp. 1847-1850, 2007.

[19] F. Camacho, P. González-Tello, E. Jurado, and A. Robles, "Microcrystalline-cellulose hydrolysis with concentrated sulphuric acid," Journal of Chemical Technology and Biotechnology, vol. 67, no. 4, pp. 350-356, 1996.

[20] M. Ioelovich, "Study of cellulose interaction with concentrated solutions of sulfuric acid," ISRN Chemical Engineering, vol. 2012, Article ID 428974, 7 pages, 2012.

[21] P. Strunk, Characterization of cellulose pulps and the influence of their properties on the process and production of viscose and cellulose ethers [Doctor dissertation], Umeå University, Umeå, Sweden, 2012.

[22] T.-C. Hsu, G.-L. Guo, W.-H. Chen, and W.-S. Hwang, "Effect of dilute acid pretreatment of rice straw on structural properties and enzymatic hydrolysis," Bioresource Technology, vol. 101, no. 13, pp. 4907-4913, 2010.

[23] D. Knappert, H. Grethlein, and A. Converse, "Partial acid hydrolysis of cellulosic materials as a pretreatment for enzymatic hydrolysis," Biotechnology and Bioengineering, vol. 22, no. 7, pp. 1449-1463, 1980.

[24] H. A. Krässig, Cellulose: Structure, Accessibility, and Reactivity, Gordon and Breach Science, 1993.

[25] A. Buleon and H. Chanzy, "Single crystals of cellulose $\mathrm{IV}_{I I}$ : preparation and properties," Journal of Polymer Science: Polymer Physics Edition, vol. 18, no. 6, pp. 1209-1217, 1980.

[26] A. D. French, "Idealized powder diffraction patterns for cellulose polymorphs," Cellulose, vol. 21, no. 2, pp. 885-896, 2014.

[27] D. Klemm, B. Heublein, H.-P. Fink, and A. Bohn, "Cellulose: fascinating biopolymer and sustainable raw material," Angewandte Chemie-International Edition, vol. 44, no. 22, pp. 3358$3393,2005$. 
[28] S. Park, J. O. Baker, M. E. Himmel, P. A. Parilla, and D. K. Johnson, "Cellulose crystallinity index: measurement techniques and their impact on interpreting cellulase performance," Biotechnology for Biofuels, vol. 3, article 10, 2010.

[29] S. R. Levis and P. B. Deasy, "Production and evaluation of size reduced grades of microcrystalline cellulose," International Journal of Pharmaceutics, vol. 213, no. 1-2, pp. 13-24, 2001.

[30] S. Beck-Candanedo, M. Roman, and D. G. Gray, "Effect of reaction conditions on the properties and behavior of wood cellulose nanocrystal suspensions," Biomacromolecules, vol. 6, no. 2, pp. 1048-1054, 2005.

[31] W. Bai, J. Holbery, and K. Li, "A technique for production of nanocrystalline cellulose with a narrow size distribution," Cellulose, vol. 16, no. 3, pp. 455-465, 2009.

[32] U. S. E. P. Agency, Solid Waste, 2013, http://www.epa.gov/ region9/waste/solid/house.html.

[33] D. Ishii, D. Tatsumi, and T. Matsumoto, "Effect of solvent exchange on the solid structure and dissolution behavior of cellulose," Biomacromolecules, vol. 4, no. 5, pp. 1238-1243, 2003.

[34] D. Ishii, D. Tatsumi, and T. Matsumoto, "Effect of solvent exchange on the supramolecular structure, the molecular mobility and the dissolution behavior of cellulose in LiCl/DMAc," Carbohydrate Research, vol. 343, no. 5, pp. 919928, 2008.

[35] A. S. Mujumdar, Handbook of Industrial Drying, CRC Press, 2006.

[36] Y. Peng, D. J. Gardner, Y. Han, A. Kiziltas, Z. Cai, and M. A. Tshabalala, "Influence of drying method on the material properties of nanocellulose I: thermostability and crystallinity," Cellulose, vol. 20, no. 5, pp. 2379-2392, 2013.

[37] F.-Y. Huang, "Thermal properties and thermal degradation of cellulose tri-stearate (CTs)," Polymers, vol. 4, no. 2, pp. 10121024, 2012. 

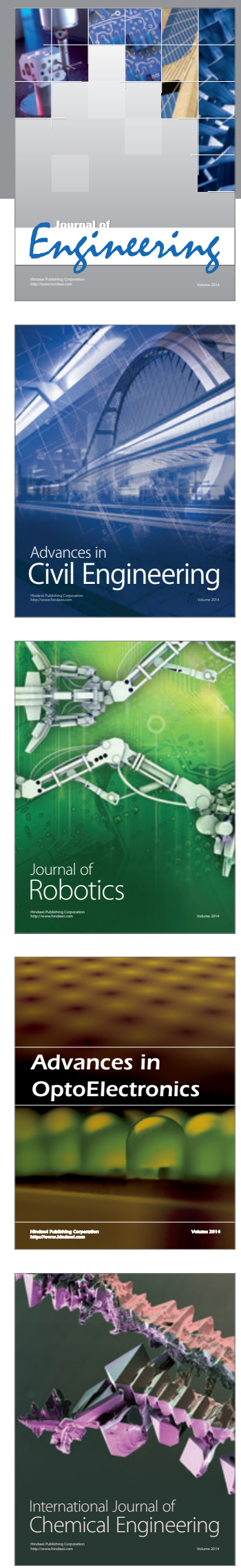

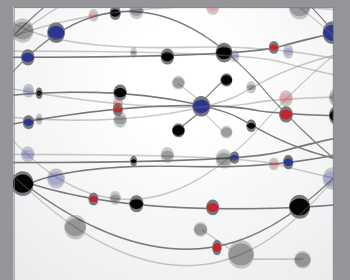

The Scientific World Journal
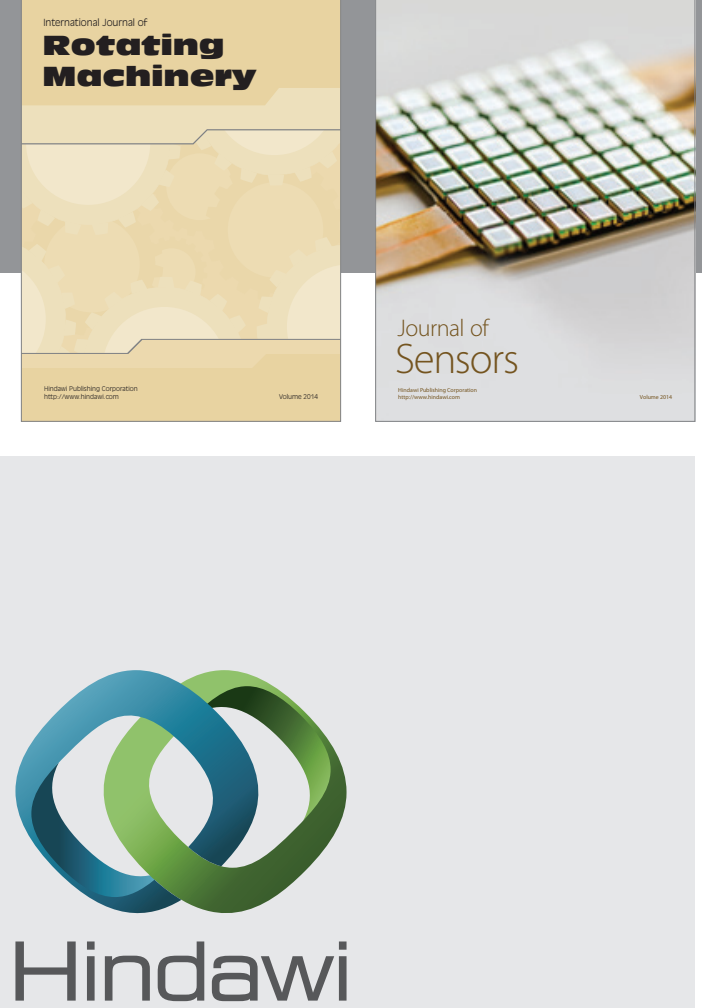

Submit your manuscripts at http://www.hindawi.com
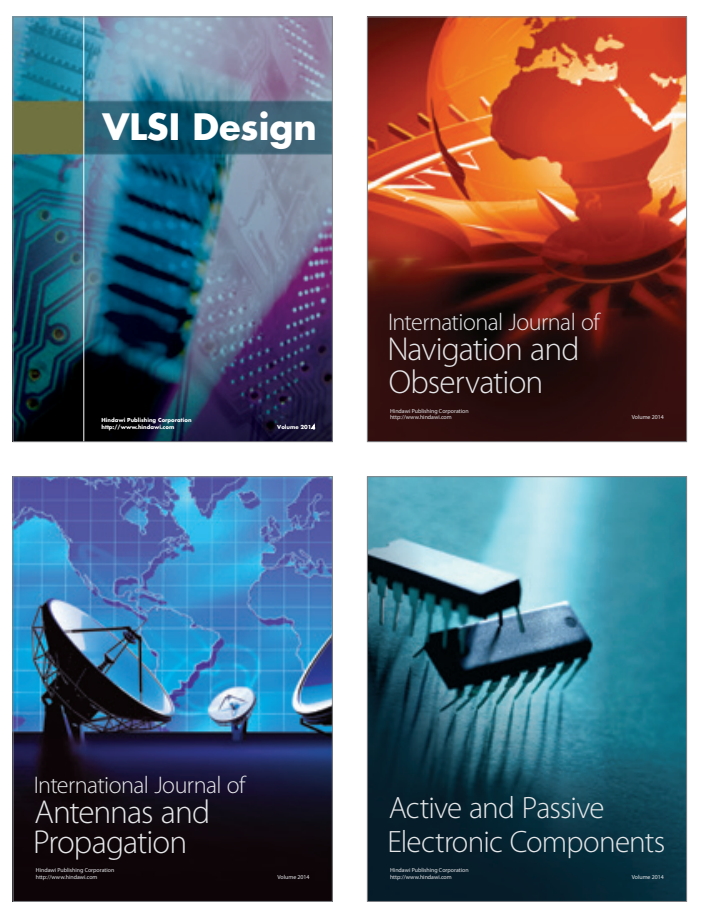
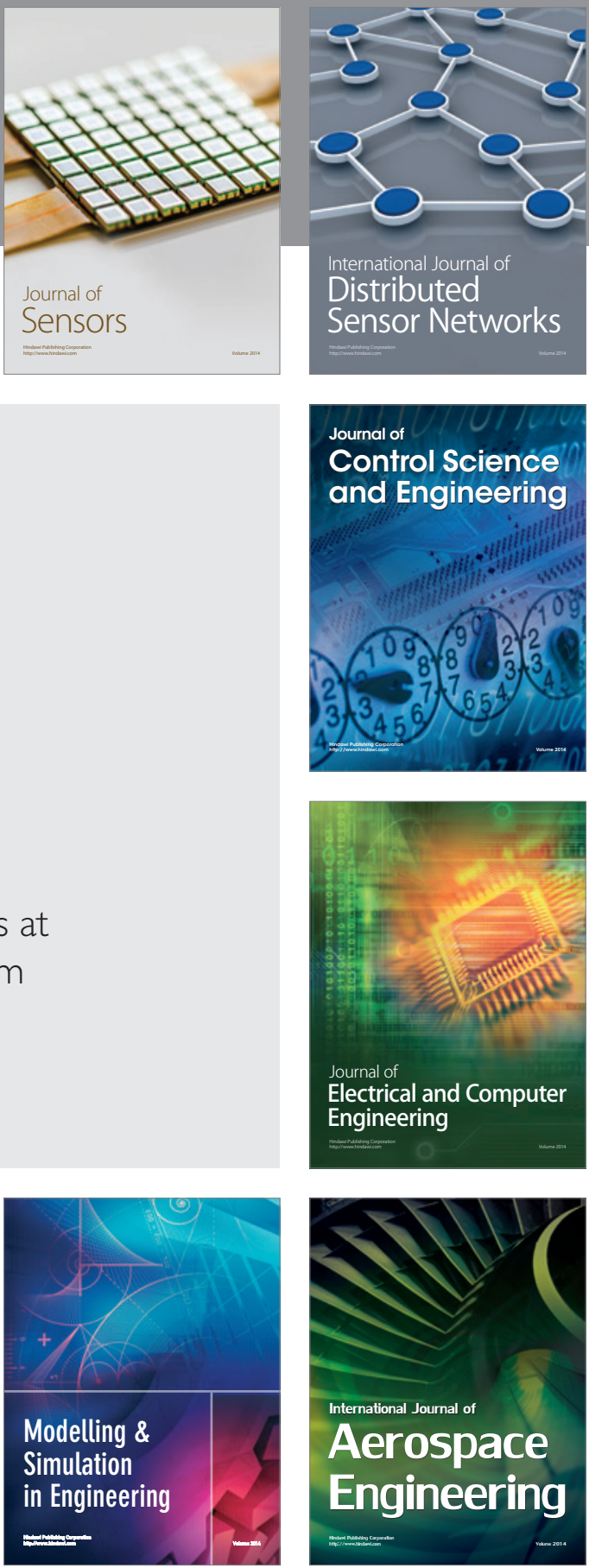

Journal of

Control Science

and Engineering
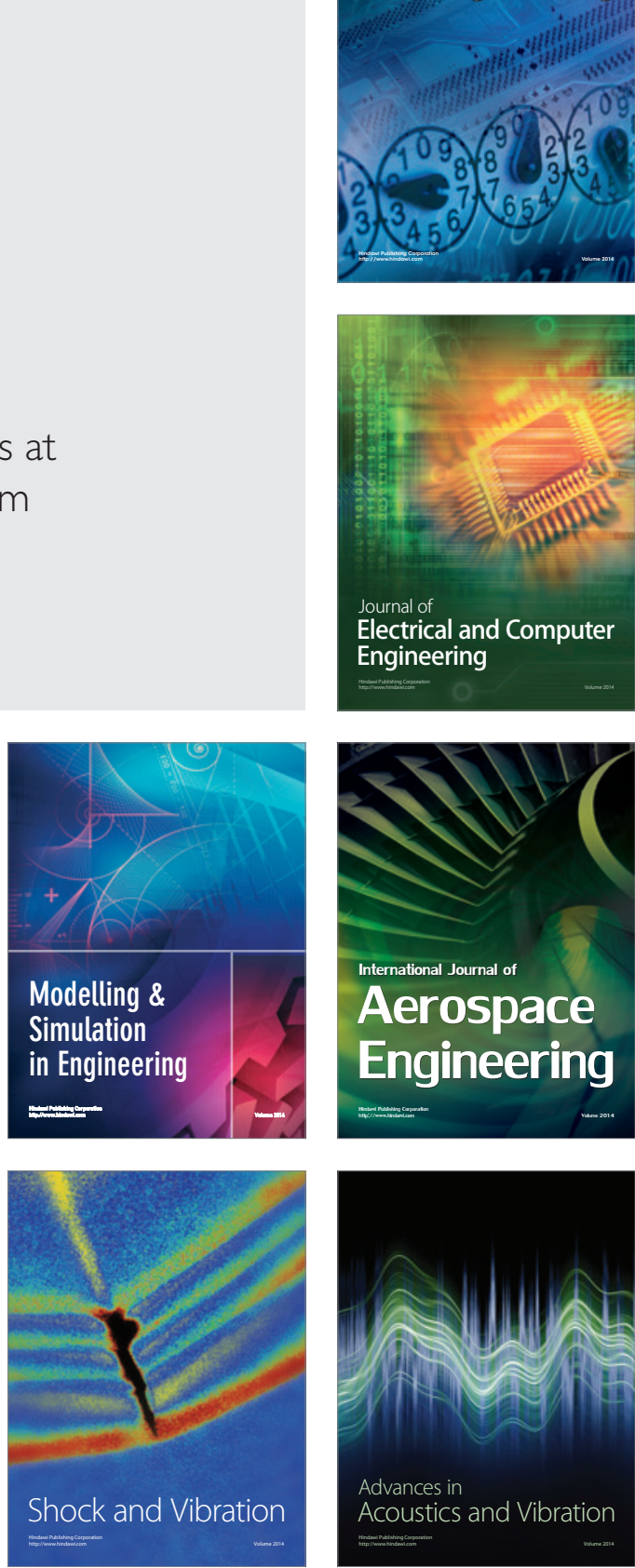